\title{
Yeast SNF2/SWI2, SNF5, and SNF6 proteins function coordinately with the gene-specific transcriptional activators GAL4 and Bicoid
}

\author{
Brehon C. Laurent and Marian Carlson \\ Department of Genetics and Development and Institute of Cancer Research, Columbia University College of Physicians and \\ Surgeons, New York, New York 10032 USA
}

The SNF2 (SWI2), SNF5, and SNF6 genes are required for transcription of many diversely regulated genes in Saccharomyces cerevisiae. Previously, we showed that SNF2, SNF5, and SNF6 function interdependently in transcriptional activation, possibly forming a heteromeric complex. Here, we present evidence that SNF6 has a more direct role in stimulating transcription than SNF2 and SNF5. The global effects of mutations in SNF2, SNF5, and SNF6 suggested that these SNF proteins may function coordinately with many gene-specific activators. We show that LexA-GAL4 and LexA-Bicoid fusion proteins are both dependent on SNF2, SNF5, and SNF6 for activation of target genes containing one or multiple lexA operators. The stringency of the requirement for the SNF proteins varies with the activator, the number of binding sites for the activator, and the target promoter. Thus, these SNF proteins appear to represent a class of intermediary proteins that facilitate transcriptional activation by gene-specific regulatory proteins.

[Key Words: Yeast; transcriptional activation; SNF proteins; GAL4; Bicoid]

Received May 15, 1992; revised version accepted July 8, 1992.

Transcription by eukaryotic RNA polymerase II is a complex process requiring basal initiation factors and enhancer-binding activator proteins. In many cases, other classes of intermediary and auxiliary proteins are also necessary for activated transcription (Lewin 1990; Roeder 1991). Some of these factors may be involved in mediating contacts between activators and the transcriptional apparatus. Others may alleviate nucleosomal repression of transcription (Grunstein 1990; Kornberg and Lorch 1991; Felsenfeld 1992).

The SNF2, SNF5, and SNF6 genes (henceforth, SNF2,5,6) of Saccharomyces cerevisiae are required for transcription of a broad spectrum of differently regulated genes. Originally identified as positive regulators of the SUC2 (invertase) gene (Neigeborn and Carlson 1984), these SNF genes also affect expression of other glucoserepressible genes (Neigeborn and Carlson 1984; Estruch and Carlson 1990; Yoshimoto and Yamashita 1991), PHO5 (Abrams et al. 1986), Ty elements (Ciriacy et al. 1991; Happel et al. 1991), cell type-specific genes (Laurent et al. 1990), and protease B (Moehle and Jones 1990). Moreover, SNF2 is the same gene as $S W I 2$ (K. Nasmyth, pers. comm.) and is therefore also required for expression of the HO and INO1 genes (Herskowitz 1989; Peterson et al. 1991). In addition, these $S N F$ genes are important for the healthy growth of the cell and for sporulation of diploids. Genetic evidence, based on interactions with extragenic suppressors, suggests that $S N F 2,5,6$ are functionally related genes (Neigeborn et al. 1986, Estruch and Carlson 1990|. Their pleiotropic mutant phenotypes indicate that $S N F 2,5,6$ have a global function in the transcriptional process.

Previous work implicated $S N F 2,5,6$ in transcriptional activation. We showed that SNF2 and SNF5 encode nuclear proteins that function as transcriptional activators in vivo (Laurent et al. 1990, 1991). Bifunctional LexASNF2 and LexA-SNF5 proteins, when tethered to DNA by a $\operatorname{lex} A$ operator, strongly activated expression of a target gene in the assay system devised by Brent and Ptashne (1985). Consistent with the genetic evidence for related functions, activation by LexA-SNF2 required both SNF5 and SNF6, and activation by LexA-SNF5 was dependent on SNF2 and SNF6 (Laurent et al. 1991). These findings indicate that the SNF2,5,6 proteins function interdependently in transcriptional activation. One possibility is that the SNF proteins form a heteromeric complex; therefore, tethering SNF2 or SNF5 near a promoter recruits the other two SNF proteins. However, the association of SNF6 with SNF2 and SNF5 was uncertain; for example, SNF6 could be required in a step before activation, such as modification of SNF2 or SNF5. In this study we further define the role of SNF6 by analysis of a LexA-SNF6 fusion protein.

We also test the dependence of LexA-SNF proteins on 
SWI1 for activation. The $S W I 1, S N F 2 / S W I 2$, and $S W I 3$ genes are related by genetic criteria (Stern et al. 1984; Sternberg et al. 1987), and Peterson and Herskowitz (1992) recently reported evidence that the five $S N F$ and $S W I$ genes are functionally related and proposed that the proteins form a multisubunit complex.

How do SNF2,5,6 affect transcriptional activation of many diversely regulated genes? The primary structures of these proteins provided few clues. None of the SNF or SWI proteins appears to provide a DNA-binding domain mediating association with different promoters (Estruch and Carlson 1990; Laurent et al. 1990, 1991; Peterson and Herskowitz 1992). The most noteworthy features are that SNF2 contains sequences resembling the motifs characteristic of helicases (Davis et al. 1992; Laurent et al. 1992), that SNF5 is strikingly rich in glutamine and proline, and that all contain acidic regions. Previously, we and others proposed that SNF2,5,6 act in conjunction with gene-specific activators to stimulate transcription (Laurent et al. 1991; Peterson and Herskowitz 1992). To test this idea, we examine here the function of two transcriptional activators, the yeast GAL4 and Drosophila Bicoid proteins, in snf2, snf5, and snf6 mutants. Using LexA-GAL4 and LexA-Bicoid fusion proteins (Brent and Ptashne 1985; Hanes and Brent 1989), we found that activation of a target gene is affected by SNF2,5,6 to varying degrees depending on the activator, the number of binding sites for the activator, and the target promoter.

How do SNF2,5,6 facilitate transcriptional activation by gene-specific activators? Our findings, coupled with previous genetic data implicating chromatin as a target of SNF function, suggest that the SNF proteins may have dual functions, both to relieve nucleosomal repression and to stimulate the transcriptional apparatus.

\section{Results}

\section{Activation by a LexA-SNF6 fusion protein}

Previously, we showed that activation by LexA-SNF2 and LexA-SNF5 requires the SNF6 function (Laurent et al. 1991). One model is that SNF2, SNF5, and SNF6 are components of a heteromeric complex. An alternative is that SNF6 acts in a step before activation, perhaps affecting the function of the SNF2 or SNF5 protein by modification; effects on protein levels, DNA binding, or nuclear localization have been excluded (Laurent et al. 1990,1991 ).

If SNF6 is part of a heteromeric complex, then tethering SNF6 to DNA might suffice to recruit SNF2, SNF5, and any other proteins that are similarly recruited by LexA-SNF2 or LexA-SNF5. According to this model, LexA-SNF6 should function as an activator. In contrast, if SNF6 modifies another SNF protein, we would not expect LexA-SNF6 to be an activator.

To test these possibilities, we constructed a $\operatorname{lexA-}$ SNF6 gene fusion in the vector pSH2-1. The SNF6 gene was fused at codon 11 to the lexA DNA-binding domain under control of the $A D H 1$ promoter (Fig. 1). The resulting plasmid, pLexA-SNF6, expresses a bifunctional

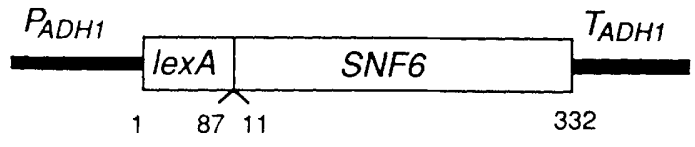

Figure 1. Expression of LexA-SNF6 fusion protein. pLexASNF6 contains the amino-terminal 87 codons of the E. coli lexA protein fused to codons 11-332 of $S N F 6$, expressed from the constitutive $A D H 1$ promoter $P_{A D H 1}$ (see Materials and methods). $\left(T_{A D H 1}\right) A D H 1$ transcriptional termination sequences.

LexA-SNF6 fusion protein, which complements a snf6s mutation. We then cotransformed wild-type yeast cells with pLexA-SNF6 and each of the lexA-GAL1-lacZ target plasmids. The target genes contain zero, one, or multiple (six overlapping; Ebina et al. 1983) lexA operators located $5^{\prime}$ to the promoter of a GAL1-lacZ fusion with a deletion of $\mathrm{UAS}_{\mathrm{G}}$ /West et al. 1984; Hanes and Brent 1989). Transcription from the GAL1 promoter is dependent on the binding of an activator protein to the lex $A$ operator (Table 1 ).

The LexA-SNF6 fusion protein activated transcription with even greater efficacy than the LexA-SNF2 and LexA-SNF5 fusions (Table 1). The ability of LexA-SNF6 to function as an activator suggests that the dependence of LexA-SNF2 and LexA-SNF5 on SNF6 does not reflect a requirement for SNF6 in a step before activation. These data are compatible with the idea that SNF6 associates with SNF2 and SNF5 in a heteromeric complex.

We then examined the dependence of LexA-SNF6 on SNF2 and SNF5 for activation of the target genes. In snf2 and snf5 mutants, expression of $\beta$-galactosidase from the target gene with one lexA operator was reduced 16- and 7-fold, respectively (Table 1). The level of LexA-SNF6 protein in these snf mutants was also reduced relative to wild type, as judged by immunoblotting (Fig. 2B), which likely contributed to the reduced activation. Thus, activation by LexA-SNF6 is at best only moderately dependent on SNF2 and SNF5. Moreover, when the target has multiple operators, activation is not at all dependent on SNF2 or SNF5 (Table 1).

In analogous assays, LexA-SNF2 and LexA-SNF5 showed striking dependence on the other SNF proteins, even when the target contained multiple lexA operators (Table 1) and the levels of LexA-SNF2 and LexA-SNF5 were not decreased in snf mutants (Laurent et al. 1991). Thus, the minimal dependence of LexA-SNF6 on SNF2 or SNF5 indicates that SNF6 is functionally distinct from SNF2 and SNF5. This finding does not exclude the possibility that SNF6 is part of a complex with SNF2 and SNF5 but suggests that SNF6 may interact more directly with components of the transcriptional apparatus.

SWIl is required for activation by LexA-SNF2 and LexA-SNF5, but not LexA-SNF6

The identity of the SNF2 and SWI2 genes (K. Nasmyth, pers. comm.) prompted us to examine the relationship of other SWI genes to SNF2,5,6. SWI1 (identical to ADR6; O'Hara et al. 1988; Peterson and Herskowitz 1992), 
Table 1. Gene activation by LexA-SNF6 in snf mutants

\begin{tabular}{|c|c|c|c|c|c|c|c|c|c|c|}
\hline \multirow{2}{*}{$\begin{array}{l}\text { Number of } \\
\text { lex } A \text { operators }\end{array}$} & \multicolumn{4}{|c|}{ LexA-SNF6 } & \multicolumn{3}{|c|}{ LexA-SNF2 } & \multicolumn{3}{|c|}{ LexA-SNF5 } \\
\hline & WT & snf2 & snf5 & snf6 & WT & $\operatorname{snf5}$ & snf6 & WT & snf2 & snf6 \\
\hline 0 & $<1$ & $<1$ & $<1$ & $<1$ & $<1$ & $<\mathrm{I}$ & $<1$ & $<1$ & $<1$ & $<1$ \\
\hline 1 & 860 & 54 & 120 & 800 & 120 & 2 & 1 & 140 & 4 & 11 \\
\hline 6 & 1320 & 1620 & 1140 & 930 & 350 & 8 & 5 & 410 & 8 & 40 \\
\hline
\end{tabular}

Target plasmids were pLR1 1,1840 , and pSH18-18, carrying lexA-GAL1-lacZ gene fusions. Values are average $\beta$-galactosidase activity for four transformants. Standard errors were $<11 \%$. Values for LexA-SNF2 and LexA-SNF5 are from Laurent et al. (1991). (WT) Wild type.

SNF2/SWI2, and SWI3 constitute a group of related genes by genetic criteria: Their mutant phenotypes and interactions with extragenic suppressors are similar (Stern et al. 1984; Sternberg et al. 1987). We therefore tested whether activation of lexA-GAL1-lacZ targets by the LexA-SNF fusion proteins is dependent on SWI1 function. Activation by LexA-SNF2 and LexA-SNF5 absolutely required SWI1. Activation by LexA-SNF6 was reduced severalfold in a swil mutant when the target promoter contained a single lexA operator but was independent of $S W I 1$ when multiple operators were provided (Table 2). Levels of the LexA-SNF2,5,6 proteins were somewhat reduced in a swil mutant (Fig. 2C), but these reductions cannot fully account for the dramatic decrease in target gene activation by LexA-SNF2 and LexA-SNF5. Thus, LexA-SNF2 and LexA-SNF5 require SWI1, but LexA-SNF6 does not. Similar experiments were attempted with a swi3 mutant, but the transformants grew too poorly for reliable assays.

\section{Transcriptional activation by LexA-GAL4 is dependent on $S N F 2,5,6$}

It has been proposed that SNF2,5,6 affect diverse genes by functioning in conjunction with gene-specific activators (Laurent et al. 1991; Peterson and Herskowitz 1992). One candidate is the GAL4 activator. Several lines of evidence indicate that the SNF2,5,6 proteins are required for $G A L$ gene transcription. Mutations in these $S N F$ genes cause growth defects on galactose (Neigeborn and Carlson 1984; Estruch and Carlson 1990), and Peterson and Herskowitz (1992) recently reported decreased ex-

Table 2. Activation by the LexA-SNF fusion proteins in a swil mutant

\begin{tabular}{lccc}
\hline \multirow{2}{*}{$\begin{array}{l}\text { Number of } \\
\text { lexA operators }\end{array}$} & LexA-SNF2 & LexA-SNF5 & LexA-SNF6 \\
\cline { 2 - 4 } & $<1$ & $<1$ & $<1$ \\
0 & 1 & 1 & 150 \\
1 & 7 & 2 & 970 \\
\hline
\end{tabular}

Target plasmids were pLR1 $\Delta 1,1840$, and pSH18-18, carrying lexA-GAL1-lac $Z$ target genes. Values are average $\beta$-galactosidase units produced in three or four independent transformants. Standard errors were $<16 \%$. pression of GAL genes in snf mutants. We have tested the effects of snf2,5,6 mutations on expression of a GAL10-lacZ gene fusion (West et al. 1984) under control of $\mathrm{UAS}_{\mathrm{G}}$, the upstream activation sequence recognized by GAL4. Under galactose-inducing conditions, $\beta$-galactosidase was reduced 10 -fold in snf2 and snf5 mutants and severalfold in the snf6 mutant (Table 3). These data suggest that SNF2,5,6 may function coordinately with GAL4.

To test this idea, we obtained a plasmid expressing a LexA-GAL4 fusion protein from the $A D H 1$ promoter, which is active in these snf mutants (Laurent et al. 1991). LexA-GAL4 has been shown to activate transcription

A

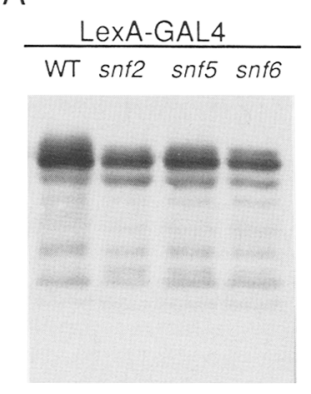

C

$\mathrm{B}$

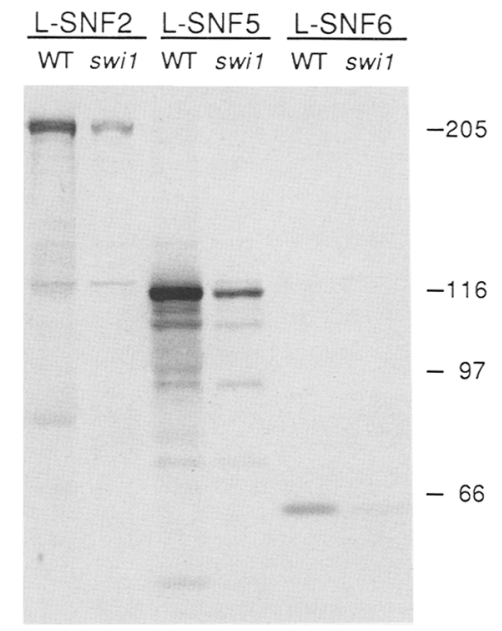

Figure 2. Immunoblot analysis of fusion proteins. Strains were MCY829 [wild type (WT)], MCY2156 (snf2), MCY2099 (snf5), MCY2101 (snf6), and CY58 (swi1) carrying the indicated expression plasmid and target plasmid 1840. Cultures were grown in SC media containing $2 \%$ glucose with selection for both plasmids. Proteins were prepared, separated by SDS-PAGE in $5 \%$ $(A), 7 \%(B)$, or $6.5 \%(C)$ polyacrylamide, and subjected to immunoblot analysis (see Materials and methods). LexA fusion proteins were detected with polyclonal LexA antiserum. Expression plasmids: $|A| \mathrm{pSH} 17-4$, expressing LexA-GAL4; $(B)$ pLexA-SNF6; $(C)$ pLexA-SNF2, pLexA-SNF5, or pLexA-SNF6, expressing LexA-SNF (L-SNF) proteins, as indicated above the lanes. Transformants used in this experiment were also assayed for $\beta$-galactosidase activity. Sizes $(\mathrm{kD})$ of marker proteins are indicated. 
Table 3. Expression of a GAL10-lacZ gene fusion in snf mutants

\begin{tabular}{|c|c|c|}
\hline Relevant genotype & Repressed & Induced \\
\hline WT & 0.2 & 650 \\
\hline$s n f 2 \Delta$ & 0.6 & 63 \\
\hline $\operatorname{snf} 5 \Delta$ & 0.2 & 69 \\
\hline $\operatorname{snf} 6 \Delta$ & 1.0 & 300 \\
\hline
\end{tabular}

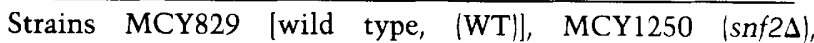
MCY1991 (snf5 5 ), and MCY2101 (snf64) carried pRY123, a $2 \mu$ plasmid containing a GAL10-lacZ fusion under the control of $\mathrm{UAS}_{\mathrm{G}}$ (West et al. 1984). For glucose-repressed cultures, the medium contained $2 \%$ glucose, $2 \%$ galactose, and $3 \%$ glycerol. For galactose induction, the medium contained $2 \%$ galactose and $3 \%$ glycerol; the snf mutants grow slowly in this medium. Values shown are average $\beta$-galactosidase activity, expressed as described by Miller (1972), for three or four individual transformants. Standard errors were $<15 \%$.

from appropriate target genes in yeast (Brent and Ptashne 1985). To determine whether the LexA-GAL4 activation function is dependent on the SNF proteins, we tested its ability to activate target gene expression in snf2, snf5, and $s n f 6$ mutant hosts. The $\operatorname{snf}$ mutations substantially reduced expression of a target gene containing one lexA operator; expression was decreased 50 -fold or more in snf2 and snf5 mutants and 16-fold in snf6 mutants (Table $3)$. In contrast, the snf mutations did not significantly affect expression from a target gene with multiple lexA operators. $\beta$-Galactosidase activities were the same within a factor of 2 in snf mutants and wild type. Levels of LexA-GAL4 protein were the same in snf mutants and wild type (Fig. 2A).

Thus, SNF2,5,6 facilitate activation by GAL4, but increasing the number of LexA-GAL4 proteins bound near the promoter compensates for loss of SNF2,5,6. These results suggest that SNF2,5,6 act coordinately with GAL4 but, in this context, are not essential intermediaries between GAL4 and the transcriptional apparatus.

\section{Dependence of the heterologous activator Bicoid on SNF function}

Bicoid, a Drosophila morphogen, is a transcriptional activator of the hunchback gene that functions in yeast but has no known yeast homolog (Hanes and Brent 1989; Struhl et al. 1989). Previously, Hanes and Brent (1989) showed that a LexA-Bicoid fusion protein activates $\operatorname{lexA}-G A L 1-l a c Z$ gene expression in yeast. We therefore tested the dependence of LexA-Bicoid on SNF2,5,6 for activation of the same target genes. Activation of a target containing one lexA operator was decreased in snf2, snf5, and snf6 mutant hosts (Table 4). Activation was strongly dependent on SNF2 and SNF5, but expression was reduced only 2.5 -fold in the snf6 mutant. In contrast to the results with LexA-GAL4, the presence of multiple lexA operators reduced, but did not abolish, the dependence on SNF2 and SNF5. Expression was reduced seven- and fourfold in snf2 and snf5 mutants, respectively.

Thus, in the case of Bicoid, which appears to be a less potent activator than GAL4 in this assay, increasing the number of LexA-Bicoid proteins bound near the promoter is not sufficient to compensate fully for loss of SNF2 or SNF5. These results confirm that the SNF proteins assist gene-specific activators in stimulating transcription. The requirement for such assistance appears to depend on the particular activator and the number of binding sites near the promoter.

\section{Requirement for SNF proteins depends on the promoter}

To determine whether the target promoter affects the dependence of an activator on the SNF proteins, we examined activation of a lexA-CYC1-lacZ target gene. Plasmid pLG670Z contains a CYC1-lacZ gene fusion with the upstream activation sequences deleted (Guarente and Ptashne 1981), and plasmid 1107 is a derivative of pLG670Z containing the lexA operator (Brent and Ptashne 1985). To confirm that the CYC1 promoter is capable of responding to SNF2,5,6, we first examined activation of these target genes by LexA-SNF5. Highlevel activation was observed in a wild-type host strain, and activation was decreased in snf2 and snf6 mutants (Table 5).

Wild-type and snf mutant strains were then cotransformed with the LexA-GAL4 or LexA-Bicoid expression plasmid and each of the target plasmids. Binding of LexA-GAL4 to one copy of the operator was sufficient for high-level activation of the CYC1 promoter in both wild-type and snf mutants (Table 5). The snf mutations caused no discernible effect, indicating that the SNF proteins do not augment the interaction of GAL4 with the transcriptional apparatus at this promoter. In contrast, activation by LexA-Bicoid was highly dependent on SNF

Table 4. Transcriptional activation by LexA-GAL4 and LexA-Bicoid fusion proteins depends on SNF proteins

\begin{tabular}{|c|c|c|c|c|c|c|c|c|}
\hline \multirow{2}{*}{$\begin{array}{l}\text { Number of } \\
\text { lex } A \text { operators }\end{array}$} & \multicolumn{4}{|c|}{ LexA-GAL4 } & \multicolumn{4}{|c|}{ LexA-Bicoid } \\
\hline & WT & snf2 & snf5 & $\operatorname{snf6}$ & WT & snf2 & $\operatorname{snf5}$ & snf6 \\
\hline 0 & $<1$ & $<1$ & $<1$ & $<1$ & $<1$ & $<1$ & $<1$ & $<1$ \\
\hline 1 & 1325 & 7 & 25 & 85 & 80 & 3 & 6 & 32 \\
\hline 6 & 1880 & 770 & 1570 & 770 & 610 & 90 & 140 & 460 \\
\hline
\end{tabular}

Target plasmids were pLR1 1,1840 , and pSH18-18, carrying lexA-GAL1-lacZ target genes. LexA-GAL4 and LexA-Bicoid were expressed from plasmids pSH17-4 and pSH11-1, respectively. Values are average $\beta$-galactosidase activity for three or four transformants. Standard errors were $<23 \%$. Control experiments with pSH2-1, expressing only the LexA DNA-binding domain, showed that the LexA moiety does not suffice for gene activation (Brent and Ptashne 1985; Laurent et al 1991). (WT) Wild type. 
Table 5. Activation of lexA-CYCl-lacZ target gene

\begin{tabular}{|c|c|c|c|c|c|c|c|c|c|c|c|c|}
\hline \multirow{2}{*}{$\begin{array}{l}\text { Number of } \\
\text { lex } A \text { operators }\end{array}$} & \multicolumn{4}{|c|}{ LexA-GAL4 } & \multicolumn{4}{|c|}{ LexA-Bicoid } & \multicolumn{3}{|c|}{ LexA-SNF5 } & \multirow{2}{*}{$\frac{\operatorname{LexA}_{1-87}}{W T}$} \\
\hline & WT & snf2 & $\operatorname{snf5}$ & snf6 & WT & $\operatorname{snf} 2$ & snf5 & snf6 & WT & snf2 & snf6 & \\
\hline 0 & $<1$ & $<1$ & 1 & 2 & $<1$ & $<1$ & $<1$ & 3 & 2 & 2 & 10 & ND \\
\hline 1 & 1260 & 1070 & 1180 & 1350 & 381 & 11 & 21 & 30 & 1440 & 34 & 156 & 2 \\
\hline
\end{tabular}

Values represent assays of one transformant carrying target plasmid pLG670Z (no lexA operator) and averages of three or four transformants carrying plasmid 1107 (one lexA operator). (WT) Wild type. (ND) Not determined. Standard errors were $<13 \%$.

function; $\beta$-galactosidase activity was reduced $35-18$, and 13-fold in $s n f 2, s n f 5$, and snf6 mutants, respectively.

Thus, the finding that SNF proteins do not augment activation by LexA-GAL4 does not reflect an intrinsic inability of the SNF proteins to affect transcription from the CYC1 promoter. Rather, the SNF proteins appear simply to be dispensable for efficient activation by GAL4 at this promoter. This evidence that LexA-GAL4, when bound at a single site, requires the SNF proteins for maximal activation of the GAL1 promoter, but not for activation of the CYC1 promoter, indicates that the role of the SNF proteins in facilitating activator function is promoter specific.

\section{Discussion}

Considerable genetic evidence indicates that the SNF2,5,6 proteins profoundly affect transcription of diversely regulated genes in vivo and are essential for healthy growth of the cell. The evidence presented here suggests that SNF2,5,6 act coordinately with various gene-specific activators to facilitate or enhance transcriptional activation. Both the LexA-GAL4 and LexABicoid proteins were dependent on SNF2,5,6 function for the activation of a GAL1-lacZ target gene with a single $\operatorname{lex} A$ operator. The ability of SNF2,5,6 to cooperate with both GAL4 and Bicoid is consistent with genetic evidence for the function of SNF2,5,6 with diverse activators in vivo. The relationship of the SNF2, 5,6 proteins to coactivators or mediators defined by biochemical experiments in vitro (for review, see Lewin 1990) is not yet clear. In these experiments, the requirement for SNF2,5,6 in the activation process depended on the specific activator (LexA-GAL4 or LexA-Bicoid), the number of binding sites for the activator (one or multiple), and the particular promoter (GAL1 or CYC1). For example, the binding of multiple LexA-GAL4 proteins by multiple operators largely overcame the requirement for SNF2,5,6 for activation of the GAL1 promoter. Perhaps this diminished dependence on SNF2,5,6 reflects saturation of the activation process. We imagine that the requirement for SNF2,5,6 for expression of genes from their chromosomal loci depends on the template structure and strength of the activator and promoter.

\section{SNF6 has a role distinct from SNF2 and SNF5}

To clarify the role of the SNF6 protein relative to SNF2 and SNF5, we expressed a bifunctional LexA-SNF6 fu- sion protein. We show that LexA-SNF6 is a transcriptional activator and functions to a considerable degree independently of SNF2 and SNF5. The minimal dependence of LexA-SNF6 on SNF2 and SNF5 /compared with the dramatic dependence of LexA-SNF2 and LexA-SNF5 on SNF6) implicates SNF6 as the protein that acts more directly to stimulate transcription. Consistent with this idea, LexA-SNF6 showed a similar pattern of dependence on $S N F 2, S N F 5$, and the number of $\operatorname{lex} A$ operators as found for LexA-GAL4 and LexA-Bicoid. Moreover, the effects of snf2,5,6 mutations on activation by LexAGAL4 and LexA-Bicoid can be understood within this context: For a target gene with a single lexA operator, the SNF6 protein plays a more modest role than SNF2 and SNF5 because its function is partially redundant with that of GAL4 and Bicoid. We note that the SNF6 protein contains both acidic and glutamine-rich regions (Estruch and Carlson 1990), which could serve as activation domains. Alternatively, SNF6 may mediate the association of yet another protein containing an activation domain.

Additional genetic evidence previously suggested that SNF6 is functionally distinct from SNF2 and SNF5. Activation by LexA-SNF2 and LexA-SNF5 was restored by an spt 6 mutation in snf5 or snf2 mutants, respectively, but was not restored in a snf6 mutant (Laurent et al. 1991). SPT6 belongs to a group of SPT genes, including genes encoding histones $\mathrm{H} 2 \mathrm{~A}$ and $\mathrm{H} 2 \mathrm{~B}$, which are thought to affect chromatin structure /Clark-Adams et al. 1988; Swanson et al. 1990).

These findings are compatible with the simple model that SNF2,5,6 form a heteromeric complex and that LexA-SNF2 or LexA-SNF5 recruits the other two SNF proteins into a DNA-bound complex (Laurent et al. 1991). (The association of SNF6 with either SNF2 or SNF5 would then require the other SNF protein because LexA-SNF2 requires SNF5 for activation, and vice versa.) The decreased levels of LexA-SNF6 protein in snf 2 and $s n f 5$ mutants are also consistent with its presence in a heteromeric complex that confers stability. However, the data do not exclude models in which SNF6 instead associates directly with the transcriptional machinery.

\section{The relationship of SWI1 to the SNF proteins}

The identity of SNF2 and SWI2 suggested that the SWI1 protein is functionally related to SNF2,5,6, and recent work of Peterson and Herskowitz (1992) provided sup- 
porting evidence. We show here that activation by LexA-SNF2 and LexA-SNF5 is abolished in a swi1 mutant, but activation by LexA-SNF6 is reduced only severalfold. We also found that levels of LexA-SNF proteins are reduced in the swi1 mutant, although transcription from the $A D H 1$ promoter is not dependent on SWIl under these growth conditions (Peterson and Herskowitz 1992). Our findings are consistent with the idea that SWIl is in a multiprotein complex with the SNF proteins, as proposed by Peterson and Herskowitz (1992), although the possibility that SWII modifies a protein in the complex (other than SNF6) has not been excluded. The observed dependency relationships rule out the model that SWII provides an activation domain that is recruited by SNF6.

We also note that SWI1, like the SNF proteins, appears to affect the function of gene-specific activators. A swi1 mutation reduces the ability of the Drosophila fushi tarazu (ftz) protein, expressed in yeast, to activate a reporter gene with upstream $\mathrm{ftz}$-binding sites, and reduces UAS $_{\mathrm{G}}$ activity in strains overexpressing GAL4 (Peterson and Herskowitz 1992).

\section{SNF2,5,6 may facilitate activation by altering chromatin}

How do the SNF2,5,6 proteins facilitate the effects of transcriptional activators? The ability of the LexA-SNF proteins to activate transcription suggests a direct stimulatory role in transcription. In addition, genetic evidence suggests that SNF2,5,6 may also affect chromatin structure. Recent work has documented nucleosomemediated repression of transcriptional initiation and changes in chromatin structure accompanying transcription (for reviews, see Grunstein 1990; Kornberg and Lorch 1991; Felsenfeld 1992). The evidence implicating SNF2,5,6 in remodeling chromatin derives from studies of extragenic suppressors that restore transcription in snf mutants. Mutations in genes encoding histones $\mathrm{H} 2 \mathrm{~A}$ and H2B (HTA1/SPT11 and HTB1/SPT12) and the related genes SPT4, SPT5, SPT6, and SPT16 suppress defects in Ty and SUC2 expression caused by snf2, snf5, and snf6 (Neigeborn et al. 1986, 1987; Clark-Adams and Winston 1987; Happel et al. 1991; Malone et al. 1991; J. Hirschhorn, S. Brown, C. Clark, and F. Winston, pers. comm.). It seems likely that mutations in these $S P T$ genes suppress the requirement for $S N F 2,5,6$ by affecting chromatin structure (Happel et al. 1991; Swanson et al. 1991). Two of the SIN genes, identified as suppressors of swi mutations that restore $H O$ transcription, also are involved in chromatin structure: SIN1 encodes an HMG1related protein (Kruger and Herskowitz 1991), and SIN2 encodes histone H3 (W. Kruger, C. Peterson, A. Sil, and I. Herskowitz, unpublished data cited by Peterson and Herskowitz 1992). This genetic evidence that changes in chromatin compensate for lack of SNF2,5,6 function strongly suggests, but does not necessarily imply, that SNF2,5,6 affect chromatin.

Alteration of chromatin by the SNF2,5,6 proteins could facilitate transcriptional activation by at least two mechanisms. One possibility is that SNF2,5,6 alter chromatin structure and facilitate contact between activators and the transcriptional apparatus, thereby enhancing stimulation of transcription. Alternatively, Workman et al. (1991) have proposed that acidic activators help basal transcription factors compete with nucleosomes for occupancy of the promoter, and SNF2,5,6 could assist this process.

We favor the idea that the SNF2,5,6 proteins function coordinately with activators in dual roles, both in alleviating nucleosomal repression and stimulating the transcriptional machinery. We suggest that SNF2 and SNF5 may be primarily responsible for disrupting or displacing nucleosomes because snf2 and snf5 mutations (but not snf6) are suppressed by spt 6 in the LexA assay system, whereas SNF6 plays a more direct role in stimulating the transcriptional machinery. Figure 3 shows a possible model; a variation in which SNF6 is not part of the complex is also tenable.

The sequence of the SNF2 protein suggests a mechanism for its function. SNF2 contains a putative NTPbinding site and other sequences related to the conserved motifs found in helicases (Davis et al. 1992; Laurent et al. 1992). A precedent for effects of a helicase on DNAprotein interactions is provided by the bacteriophage $\mathrm{T} 4$ dda protein, a helicase that removes proteins from DNA (Bonne-Andrea et al. 1990). We also note that a helicase activity was found to associate with the human RAP30/ 74 (TFIIF) factor (Sopta et al. 1989).

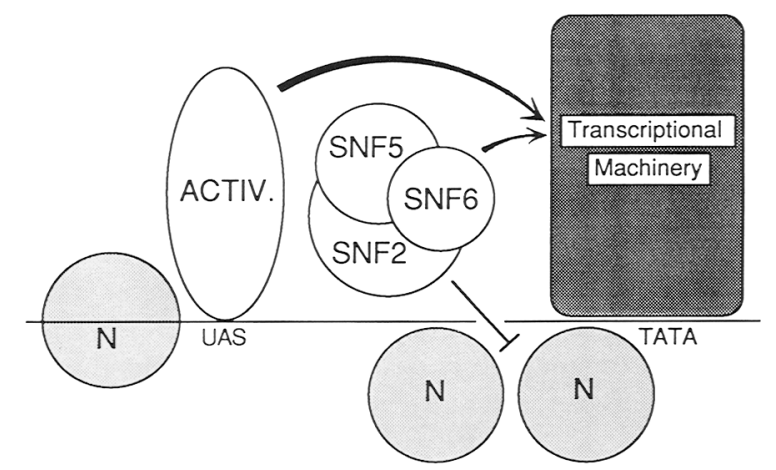

Figure 3. Model for the roles of the SNF2, SNF5, and SNF6 proteins in transcriptional activation. The proposed heteromeric complex containing SNF2, SNF5, and SNF6 may include more than one subunit of each SNF protein and also other proteins, such as SWI1 and SWI3. A possible variation of this model would depict SNF6 as a separate entity, not associated with other SNF proteins. The effect of the SNF proteins on chromatin structure is represented by a bar and a change in placement of nucleosomes ( $\mathrm{N}$, shaded circles). The SNF proteins may either disrupt or displace nucleosomes, thereby alleviating their repressive effect on transcription. Arrows indicate activation of the transcriptional machinery by a gene-specific activator protein (ACTIV.) bound to the upstream activating sequence (UAS) and by SNF6. The activator protein may also be directly involved in relieving repression by nucleosomes (Workman et al. 1991). The association of SNF2,5,6 with the promoter could be mediated by the activator, by basal transcription factors, or by an unidentified DNA-binding protein. 
SNF2,5,6 functions may be conserved among eukaryotes

Yeast and higher eukaryotes show a high degree of conservation for the basic processes of transcription, and many activators and transcription factors function interchangeably in yeast and mammalian cells (Kelleher et al. 1992 and references therein). The SNF2,5,6 proteins not only function globally in yeast but also assist the heterologous activator Bicoid, suggesting conservation of SNF function in evolution. Proteins with extensive homology to SNF2 have already been identified in Drosophila, Bombyx mori, mouse, and human cells (Laurent et al. 1992; Tamkun et al. 1992; N. Spoerel; R. Perry and V. Delmas; R. Nussbaum; all pers. comm.l, and the Drosophila homolog Brahma appears to be involved in transcriptional activation (Tamkun et al. 1992). Thus, it seems highly likely that the SNF2,5,6 proteins have functional counterparts in other eukaryotes.

\section{Materials and methods}

Strains, media, and genetic methods

All S. cerevisiae strains were derivatives of $\mathrm{S} 288 \mathrm{C}$ and are listed in Table 6. Strains MCY829, MCY2156, MCY2099, and MCY2101 were used as hosts unless otherwise indicated. Rich (YPD) and synthetic complete (SC) media (Rose et al. 1990) contained $2 \%$ glucose, except where noted otherwise. Transformation and genetic analysis of yeast were by standard methods (Rose et al. 1990). Escherichia coli strains HB101 and XL-1 Blue were used as hosts for plasmids.

\section{Plasmids}

Expression plasmids $\mathrm{pSH} 2-1$, a $2 \mu$-based plasmid bearing the HIS3 selectable marker, expresses the amino-terminal 87 residues of the LexA protein $\left(\operatorname{LexA}_{1-87}\right)$ from the constitutive $A D H 1$ promoter (Hanes and Brent 1989). The following LexA-fusion plasmids are derivatives of $\mathrm{pSH} 2-1$ and express $\mathrm{LexA}_{1-87}$ fused to the indicated sequence: pLexA-SNF2, SNF2 residues 14-1696 (Laurent et al. 1991); pLexA-SNF5, SNF5 residues 9-868 (Laurent et al. 1990); pSHl1-1, Drosophila Bicoid residues 3-489 (Hanes and Brent 1989); and pSH17-4, residues 74 881 of GAL4 (S.D. Hanes, pers. comm.).

To construct pLexA-SNF6 (Fig. 1), we isolated the 0.9-kb Ncol fragment of pEB107 (F. Estruch, unpubl.), a subclone of the NheI-Xhol fragment of pEL3.10 (Estruch and Carlson 1990) in

Table 6. List of S. cerevisiae strains

\begin{tabular}{|c|c|}
\hline Strain & Genotype \\
\hline MCY829 & MAT $\alpha$ his3A200 lys2-801 ura3-52 SUC2 \\
\hline MCY1250 & $\begin{array}{l}\text { MATa snf2 } 1:: H I S 3 \text { his } 3 \Delta 200 \text { lys2-801 ura3-52 } \\
\text { SUC2 }\end{array}$ \\
\hline MCY1991 & MATa snf5 $\Delta 2$ ura3-52 ade2-101 SUC2 \\
\hline MCY2099 & 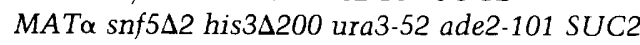 \\
\hline MCY2101 & MATa snf6A2 his $3 \Delta 200$ ura3-52 lys2-801 SUC2 \\
\hline MCY2156 & MATa snf2-141 oc his $3 \Delta 200$ ura3-52 SUC2 \\
\hline CY58 ${ }^{\mathrm{a}}$ & $\begin{array}{l}\text { MATa swi1 } 1: \text { LEU2 his } 3 \Delta 200 \text { lys2-801 ura3-52 } \\
\text { ade2-101 trp1 } 1 \text { leu2- } \Delta 1\end{array}$ \\
\hline
\end{tabular}

abtained from C. Peterson and I. Herskowitz.
pUC19. After the ends were made flush with the Klenow fragment of DNA polymerase I, the fragment was ligated to BamHI 10 -mer linkers and cloned into the BamHI site of pSH2-1. The resulting plasmid, pLexA-SNF6, encodes LexA $A_{1-87}$ fused inframe to codons 11-332 of SNF6. The pSH2-1 polylinker and BamHI linker introduce 8 residues between LexA and SNF6. The SNF6 translational stop codon follows residue 332.

Target plasmids The reporter plasmids contain the $2 \mu$ replicator and the URA3 selectable marker. pLRIDl (West et al. 1984) contains a GAL1-lacZ gene fusion, with the $U_{A} S_{G}$ removed. Plasmids 1840 [(identical to 1145 in Brent and Ptashne [1985)], and pSH18-18 (S.D. Hanes and R. Brent, pers. comm.) are derived from pLR $1 \Delta 1$ and contain a single 22-bp lexA operator or $6 \operatorname{colE} 1$ overlapping lexA operators (Ebina et al. 1983), respectively, inserted 167 bp upstream of the transcriptional start site for GAL1-lacZ. pLG670Z /Guarente and Ptashne 1981) carries a CYC1-lacZ gene fusion lacking the upstream activation sites $\mathrm{UAS}_{\mathrm{Cl}}$ and $\mathrm{UAS}_{\mathrm{C} 2}$. Plasmid 1107, originally named 1155 (Brent and Ptashne 1985), is a derivative of pLG670Z in which a single lexA operator was inserted at the Xhol site 178 bp $5^{\prime}$ to the most upstream $C Y C 1$ transcription start site.

\section{$\beta$-Galactosidase assays}

Freshly transformed colonies were grown to mid-log phase in SC medium lacking appropriate supplements to maintain selection for plasmids and containing $2 \%$ glucose except where noted. $\beta$-Galactosidase activity was assayed in permeabilized cells (Guarente 1983) and is expressed as described by Miller (1972). Four (occasionally three) transformants of each genotype were assayed in each experiment. In control experiments to test the effects of carbon source on the activation assay, wild-type cultures carrying pLexA-SNF5 and 1840 were shifted to medium containing $2 \%$ raffinose for $3 \mathrm{hr}$; this shift to nonrepressing conditions did not increase $\beta$-galactosidase expression significantly.

\section{Immunoblot analysis}

Protein extracts were prepared as described (Celenza and Carlson 1986), except that pepstatin and leupeptin were each added to a final concentration of $1 \mu \mathrm{g} / \mathrm{ml}$, and lysates were clarified by centrifuging at $10,000 \mathrm{~g}$ for $10 \mathrm{~min}$. Proteins were separated by SDS-PAGE and electroblotted to nitrocellulose paper as described (Towbin et al. 1979) except that methanol was omitted. Blots were incubated with polyclonal LexA antibody (a gift of J. Kamens and R. Brent, Massachusetts General Hosp., Boston) at a dilution of $1: 2000$ followed by goat anti-rabbit antibody coupled to alkaline phosphatase (Promega). Reactions were developed with the NBT/BCIP color reagents (Promega).

\section{Acknowledgments}

We thank Kwan Choi for assistance with experiments, Steve Hanes, Joanne Kamens, and Roger Brent for generous gifts of plasmids and antibody, and Craig Peterson and Ira Herskowitz for providing the swil mutant. We thank Isabelle Treich and Aaron Mitchell for helpful discussion. This work was supported by grants GM34095 and GM47259 from the National Institutes of Health and by an American Cancer Society Faculty Research Award to M.C. B.C.L. acknowledges support from National Research Service Award Postdoctoral Fellowship F32 GM11933.

The publication costs of this article were defrayed in part by payment of page charges. This article must therefore be hereby 
marked "advertisement" in accordance with 18 USC section 1734 solely to indicate this fact.

\section{References}

Abrams, E., L. Neigeborn, and M. Carlson. 1986. Molecular analysis of $S N F 2$ and $S N F 5$, genes required for expression of glucose-repressible genes in S. cerevisiae. Mol. Cell. Biol. 6: 3643-3651.

Bonne-Andrea, C., M.L. Wong, and B.M. Alberts. 1990. In vitro replication through nucleosomes without histone displacement. Nature 343: 719-726.

Brent, R. and M. Ptashne. 1985. A eukaryotic transcriptional activator bearing the DNA specificity of a prokaryotic repressor. Cell 43: 729-736.

Celenza, J.L. and M. Carlson. 1986. A yeast gene that is essential for release from glucose repression encodes a protein kinase. Science 233: 1175-1180.

Ciriacy, M., K. Freidel, and C. Lohning. 1991. Characterization of trans-acting mutations affecting $\mathrm{Ty}$ and Ty-mediated transcription in Saccharomyces cerevisiae. Curr. Genet. 20: $441-448$.

Clark-Adams, C.D. and F. Winston. 1987. The SPT6 gene is essential for growth and is required for $\delta$-mediated transcription in Saccharomyces cerevisiae. Mol. Cell. Biol. 7: 679686.

Clark-Adams, C.D., D. Norris, M.A. Osley, J.S. Fassler, and F. Winston. 1988. Changes in histone gene dossage alter transcription in yeast. Genes \& Dev. 2: 150-159.

Davis, J.L., R. Kunisawa, and J. Thorner. 1992. A presumptive helicase (MOT1 gene product) affects gene expression and is required for viability in the yeast Saccharomyces cerevisiae. Mol. Cell. Biol. 12: 1879-1892.

Ebina, Y., Y. Takahara, F. Kishi, A. Nakazawa, C. Parker, and R. Brent. 1983. LexA protein is repressor of the colicin E1 gene. I. Biol. Chem. 258: 13258-13261.

Estruch, F. and M. Carlson. 1990. SNF6 encodes a nuclear protein that is required for expression of many genes in Saccharomyces cerevisiae. Mol. Cell. Biol. 10: 2544-2553.

Felsenfeld, G. 1992. Chromatin as an essential part of the transcriptional mechanism. Nature 355: 219-224.

Grunstein, M. 1990. Nucleosomes: Regulators of transcription. Trends Genet. 6: 395-400.

Guarente, L. 1983. Yeast promoters and lacZ fusions designed to study expression of cloned genes in yeast. Methods Enzymol. 101: 181-191.

Guarente, L. and M. Ptashne. 1981. Fusion of Escherichia coli lacZ to the cytochrome c gene of Saccharomyces cerevisiae. Proc. Natl. Acad. Sci. 78: 2199-2203.

Hanes, S.D. and R. Brent. 1989. DNA specificity of the bicoid activator protein is determined by homeodomain recognition helix residue 9. Cell 57: 1275-1283.

Happel, A.M., M.S. Swanson, and F. Winston. 1991. The SNF2, SNF5, and SNF6 genes are required for Ty transcription in Saccharomyces cerevisiae. Genetics 128: 69-77.

Herskowitz, I. 1989. A regulatory hierarchy for cell specialization in yeast. Nature 342: 749-757.

Kelleher, R.J. III, P.M. Flanagan, D.I. Chasman, A.S. Ponticelli, K. Struhl, and R.D. Kornberg. 1992. Yeast and human TFIIDs are interchangeable for the response to acidic transcriptional activators in vitro. Genes \& Dev. 6: 296-303.

Kornberg, R.D. and Y. Lorch. 1991. Irresistible force meets immovable object: Transcription and the nucleosome. Cell 67: 833-836.

Kruger, W. and I. Herskowitz. 1991. A negative regulator of $H O$ transcription, SIN1 (SPT2), is a nonspecific DNA-binding protein related to HMG1. Mol. Cell. Biol. 11: 4135-4146.

Laurent, B.C., M.A. Treitel, and M. Carlson. 1990. The SNF5 protein of Saccharomyces cerevisiae is a glutamine- and proline-rich transcriptional activator that affects expression of a broad spectrum of genes. Mol. Cell. Biol. 10: 5616-5625.

Laurent, B.C., M.A. Treitel, and M. Carlson. 1991. Functional interdependence of the yeast SNF2, SNF5, and SNF6 proteins in transcriptional activation. Proc. Natl. Acad. Sci. 88: 2687-2691.

Laurent, B.C., X. Yang, and M. Carlson. 1992. An essential Saccharomyces cerevisiae gene homologous to SNF2 encodes a helicase-related protein in a new family. Mol. Cell. Biol. 12: 1893-1902.

Lewin, B. 1990. Commitment and activation at Pol II promoters: A tail of protein-protein interactions. Cell 61: 11611164.

Malone, E.A., C.D. Clark, A. Chiang, and F. Winston. 1991. Mutations in SPT16/CDC68 suppress cis- and trans-acting mutations that affect promoter function in Saccharomyces cerevisiae. Mol. Cell. Biol. 11: 5710-5717.

Miller, J.H. 1972. Experiments in molecular genetics. Cold Spring Harbor Laboratory, Cold Spring Harbor, New York.

Moehle, C. M. and E.W. Jones. 1990. Consequences of growth media, gene copy number, and regulatory mutations on the expression of the PRB1 gene of Saccharomyces cerevisiae. Genetics 124: 39-55.

Neigeborn, L. and M. Carlson. 1984. Genes affecting the regulation of SUC2 gene expression by glucose repression in Saccharomyces cerevisiae. Genetics 108: 845-858.

Neigeborn, L., I.L. Celenza, and M. Carlson. 1987. SSN20 is an essential gene with mutant alleles that suppress defects in SUC2 transcription in Saccharomyces cerevisiae. Mol. Cell. Biol. 7: 672-678.

Neigeborn, L., K. Rubin, and M. Carlson. 1986. Suppressors of snf2 mutations restore invertase derepression and cause temperature-sensitive lethality in yeast. Genetics 112: 741753.

O'Hara, P.J., H. Horowitz, G. Eichinger, and E.T. Young. 1988 The yeast $A D R 5$ gene encodes homopolymeric amino acid sequences and a potential metal-binding domain. Nucleic Acids Res. 16: 10153-10169.

Peterson, C.L. and I. Herskowitz. 1992. Characterization of the yeast $S W I 1, S W I 2$, and $S W I 3$ genes, which encode a global activator of transcription. Cell 68: 573-583.

Peterson, C.L., W. Kruger, and I. Herskowitz. 1991. A functional interaction between the C-terminal domain of RNA polymerase II and the negative regulator SIN1. Cell 64: 11351143.

Roeder, R.G. 1991. The complexities of eukaryotic transcription initiation: Regulation of preinitiation complex assembly. Trends Biochem. Sci. 16: 402-408.

Rose, M.D., F. Winston, and P. Hieter. 1990. Methods in yeast genetics: A laboratory course manual. Cold Spring Harbor Laboratory Press, Cold Spring Harbor, New York.

Sopta, M., Z.F. Burton, and J. Greenblatt. 1989. Structure and associated DNA-helicase activity of a general transcription initiation factor that binds to RNA polymerase II. Nature 341: $410-414$.

Stern, M.J., R.E. Jensen, and I. Herskowitz. 1984. Five SWI genes are required for expression of the $\mathrm{HO}$ gene in yeast. $/ . \mathrm{Mol}$. Biol. 178: 853-868.

Sternberg, P.W., M.J. Stern, I. Clark, and I. Herskowitz. 1987. Activation of the yeast $H O$ gene by release from multiple negative controls. Cell 48: 567-577.

Struhl, G., K. Struhl, and P.M. Macdonald. 1989. The gradient 
morphogen bicoid is a concentration-dependent transcriptional activator. Cell 57: 1259-1273.

Swanson, M.S., M. Carlson, and F. Winston. 1990. SPT6, an essential gene that affects transcription in Saccharomyces cerevisiae, encodes a nuclear protein with an extremely acidic amino terminus. Mol. Cell. Biol. 10: 4935-4941.

Swanson, M.S., E.A. Malone, and F. Winston. 1991. SPT5, an essential gene important for normal transcription in Saccharomyces cerevisiae, encodes an acidic nuclear protein with a carboxy-terminal repeat. Mol. Cell. Biol. 11: 3009-3019.

Tamkun, J.W., R. Deuring, M.P. Scott, M. Kissinger, A.M. Pattatucci, T.C. Kaufman, and J.A. Kennison. 1992. brahma: A regulator of Drosophila homeotic genes structurally related to the yeast transcriptional activator SNF2/SWI2. Cell 68: $561-572$.

Towbin, H., T. Staehelin, and I. Gordon. 1979. Electrophoretic transfer of proteins from polyacrylamide gels to nitrocellulose sheets: Procedure and some applications. Proc. Natl. Acad. Sci. 76: 4350-4354.

West, R.W. Jr., R.R. Yocum, and M. Ptashne. 1984. Saccharomyces cerevisiae GAL1-GAL10 divergent promoter region: Location and function of the upstream activating sequence $\mathrm{UAS}_{\mathrm{G}}$. Mol. Cell. Biol. 4: 2467-2478.

Workman, J.L., I.C.A. Taylor, and R.E. Kingston. 1991. Activation domains of stably bound GAL4 derivatives alleviate repression of promoters by nucleosomes. Cell 64: 533-544.

Yoshimoto, H. and I. Yamashita. 1991. The GAM1/SNF2 gene of Saccharomyces cerevisiae encodes a highly charged nuclear protein required for transcription of the STA1 gene. Mol. Gen. Genet. 228: 270-280. 


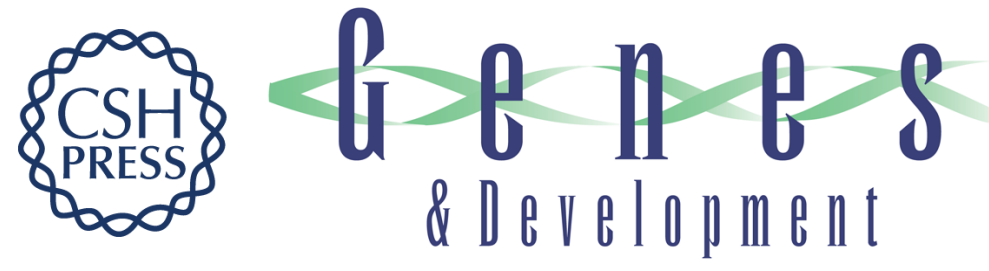

\section{Yeast SNF2/SWI2, SNF5, and SNF6 proteins function coordinately with the gene-specific transcriptional activators GAL4 and Bicoid.}

B C Laurent and M Carlson

Genes Dev. 1992, 6:

Access the most recent version at doi:10.1101/gad.6.9.1707

References This article cites 44 articles, 23 of which can be accessed free at: http://genesdev.cshlp.org/content/6/9/1707.full.html\#ref-list-1

License

Email Alerting

Service

Receive free email alerts when new articles cite this article - sign up in the box at the top right corner of the article or click here.

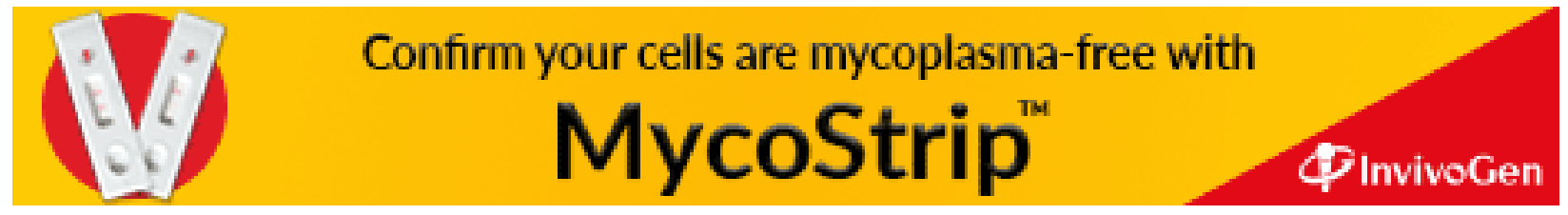

